Elsevier Editorial system(tm) for

Biochemical and Biophysical Research Communications

Manuscript Draft

Manuscript Number:

Title: Caspase-3/ROCK1 pathway mediates high glucose-induced platelet microparticles shedding

Article Type: Short communication/Full length article

Keywords: platelet microparticles; high glucose; Rho-associated kinase 1; caspase-3

Corresponding Author: Professor Kun Ling Ma, MD, PhD

Corresponding Author's Institution: Institute of Nephrology, Southeast University

First Author: Gui Hua Wang

Order of Authors: Gui Hua Wang; Kun Ling Ma, MD, PhD; Yang Zhang; Ze Bo Hu; Jian Lu; Liang Liu; Pei Pei Chen; Chen Chen Lu; Xiong Zhong Ruan; Bi Cheng Liu 


\section{Cover Letter}

Dear editorial board of Biochem Biophys Res Commun,

Please find enclosed the manuscript: Caspase-3/ROCK1 pathway mediates high glucose-induced platelet microparticles shedding by Kun Ling Ma, et al. to be submitted as a Full length article to Biochem Biophys Res Commun for consideration of publication. All co-authors have read the contents of the manuscript and there is no financial interest to report. We certify that the submission is original work and neither the manuscript nor part of it has been published or is currently under consideration for publication with any other journal.

Platelet microparticles (PMPs) are closely associated with diabetic macrovascular complications. This study aimed to explore the underlying mechanisms of high glucose-induced PMPs generation. High glucose enhanced PMPs shedding under the background of collagen. The mRNA and protein levels of ROCK1, but not ROCK2, were increased in platelets incubated with high glucose. Y-27632, an inhibitor of ROCK, blocked the increased PMPs shedding induced by high glucose. Expression and activity of caspase-3 were elevated in platelets under the high glucose conditions. Z-DVED-FMK, a caspase-3 inhibitor, inhibited ROCK1 activity and decreased the PMPs generation under high glucose. High glucose increased PMPs shedding via caspase-3/ROCK1 signal pathway. 
We hope that the editorial board will agree on the interest of this study. All the figures and tables are from the results of our own. We declare that all co-authors have read the manuscript and approved submission.

Sincerely yours,

Kun Ling Ma on behalf of the authors

Corresponding author: Kun Ling Ma at Institute of Nephrology, Zhong Da Hospital, Southeast University School of Medicine, Nanjing City, Jiangsu Province, P.R.China, 210009. E-mail address: klma05@163.com 


\section{Highlights:}

1. High glucose enhanced PMPs shedding.

2. Caspase-3/ROCK1 mediated PMPs generation under high glucose. 


\section{Caspase-3/ROCK1 pathway mediates high glucose-induced platelet microparticles shedding}

Gui Hua Wang ${ }^{1}$ MD; Kun Ling Ma ${ }^{1, a}$ MD, PhD; Yang Zhang ${ }^{1}$ MD; Ze Bo Hu${ }^{1}$ MD; Liang Liu ${ }^{1}$ MD; Jian $\mathrm{Lu}^{1}$; Pei Pei Chen ${ }^{1}$; Chen Chen Lu' ${ }^{1}$ Xiong Zhong Ruan ${ }^{2}$ PhD; Bi Cheng Liu ${ }^{1} \mathrm{MD}, \mathrm{PhD}$

${ }^{1}$ Institute of Nephrology, Zhongda Hospital, School of Medicine, Southeast University, Nanjing, 210009 , China;

${ }^{2}$ Centre for Nephrology, University College London (UCL) Medical School, Royal Free Campus, UK.

\section{${ }^{\text {a }}$ Correspondence to:}

Kun Ling Ma

Institute of Nephrology,

Zhong Da Hospital, School of Medicine, Southeast University,

NO. 87, Ding Jia Qiao Road,

Nanjing City, Jiangsu Province, China, 210009.

Tel.: 00862583262442

Email: klma05@163.com 


\begin{abstract}
Platelet microparticles (PMPs) are closely associated with diabetic macrovascular complications. This study aimed to explore the underlying mechanisms of high glucose-induced PMPs generation. Washed platelets, obtained from the plasma of healthy male Sprague-Dawley rats, were incubated with high glucose. PMPs were isolated using gradient centrifugation and counted by flow cytometry. Expression and activity of ROCK1 and caspase-3 were evaluated by real-time PCR, Western blotting, and activity assay kit. The results showed that high glucose enhanced PMPs shedding following the background of collagen. The mRNA and protein levels of ROCK1, but not ROCK2, were increased in platelets incubated with high glucose. Y-27632, an inhibitor of ROCK, blocked the increased PMPs shedding induced by high glucose. Expression and activity of caspase-3 were elevated in platelets under the high glucose conditions. Z-DVED-FMK, a caspase-3 inhibitor, inhibited ROCK1 activity and decreased the PMPs generation under high glucose. In conclusion, high glucose increased PMPs shedding via caspase-3ROCK1 signal pathway.
\end{abstract}

Keywords: platelet microparticles; high glucose; Rho-associated kinase 1; caspase-3 


\section{Introduction}

Diabetes mellitus (DM) has higher risk of cardiovascular mortality than the general population despite efforts to improve the treatments[1,2]. However, the pathogenesis of diabetic vascular complications is still unclear. It is well-known that platelet activation plays pivotal role in thrombosis and haemostasis which contributes to the progression of atherosclerosis in DM. Activated platelets possess proinflammatory and pro-coagulant function primarily through the release of platelet microparticles (PMPs). PMPs, initially described as "platelet dust", are vesicles typically around $0.1-1 \mu \mathrm{m}$ in diameter released by activated platelets[3]. The formation of PMPs are confirmed as the budding of vesicles from platelets membrane thus carrying the surface proteins of platelets. PMPs have been considered as a new mediator of intercellular communication exerting effects on inflammation, coagulation and biological information transmission[4-6]. Recently, accumulating evidence shows that plasma PMPs from diabetic patients are significantly increased and has been considered to be associated with the development of diabetic vascular complications[7-9]. Therefore, it's important to elucidate the mechanism of PMPs generation for discovering new drug targets and preventing the progression of DM mediated by platelet activation.

The generation of PMPs is primarily due to the activation of platelets by soluble agonist or shear stress[10]. Since hyperglycemia is the most common manifestation of DM, there is a strong link between impaired glycemic control and platelet activation[11]. Flow cytometry and ultrastructure analysis both confirmed that DM patients with cardiovascular complications have elevated platelet activation than DM without cardiovascular events[12]. The formation of PMPs is the process of platelet blebbing during activation. 
The membrane blebbing depends on the cytoskeleton remodeling, which are highly regulated by Rhoassociated kinase (ROCK)[13]. ROCK is an important serine/threonine protein kinase in mammalian cells. ROCK1 and ROCK2 are the two identified mammalian ROCK homologs, and their activity depends on the cleavage mediated by caspases[14]. Y-27632, a potent ROCK inhibitor, reverses membrane bleb formation[15], indicating the possible role of ROCK in microparticle generation.

Therefore, this study aimed to investigate whether high glucose induced PMPs generation and explore the potential mechanisms mediated by the caspase-ROCK pathway in the PMPs shedding.

\section{Materials and Methods}

\section{Platelet isolation}

All animals received human care. The experimental protocols were complied with the National Institutes of Health guide for the care and use of laboratory animals and approved by the Ethics Committee of Southeast University. Platelet isolation was conducted as previously described[16]. Male Sprague-Dawley rats ( 8 weeks old and 230 240 g body weight) were humanely killed and the blood was withdrawn by cardiac puncture using acid-citrate-dextrose as the anticoagulant. Platelet-rich plasma (PRP) was obtained after centrifugation at $282 \mathrm{~g}$ for 10 minutes and then at $400 \mathrm{~g}$ for 5 minutes at room temperature. After centrifuging PRP at $1600 \mathrm{~g}$ for 5 minutes, the deposition was resuspended in citrate-glucose-saline (pH 6.5) buffer. Undergoing the last centrifugation at $1600 \mathrm{~g}$ for 5 minutes, platelets were resuspended in modified Tyrode's buffer (MTB, $12 \mathrm{mmol} / \mathrm{L} \mathrm{NaHCO3,} 138 \mathrm{mmol} / \mathrm{L} \mathrm{NaCl}, 5.5 \mathrm{mmol} / \mathrm{L}$ glucose, 2.9 
$\mathrm{mmol} / \mathrm{L} \mathrm{KCl}, 0.42 \mathrm{mmol} / \mathrm{L}, \mathrm{NaH}_{2} \mathrm{PO} 4,10 \mathrm{mmol} / \mathrm{L} \mathrm{N}$-2-hydroxyethylpiperazine-N-2-ethanesulfonic acid (HEPES), $\mathrm{pH}$ 7.4) containing $1 \mathrm{mmol} / \mathrm{L} \mathrm{CaCl} 2$ and $1 \mathrm{mmol} / \mathrm{L} \mathrm{MgCl}_{2}$. Platelets were counted using Cellometer XE-2100 (Sysmex) and adjusted at $1 \times 10^{8} / \mathrm{ml}$.

\section{Experimental design for platelets in vitro}

Platelets were stimulated with $5 \mu \mathrm{g} / \mathrm{ml}$ collagen (collagen I, Sigma) for 30 minutes at $37^{\circ} \mathrm{C}$ under steady stirring with or without glucose $(25 \mathrm{mmol} / \mathrm{L})$ pre-treatment for 10 minutes. Mannitol $(25 \mathrm{mmol} / \mathrm{L})$ was used as the osmotic pressure control. The final concentration of glucose was $30 \mathrm{mmol} / \mathrm{L}$ whereas it was 5 $\mathrm{mmol} / \mathrm{L}$ in the control. For the study of PMPs-shedding mechanism, washed platelets were pre-treated with ROCK inhibitor, Y-27632 (10 $\mu \mathrm{mol} / \mathrm{L}$; Absin Bioscience), or a specific caspase-3 inhibitor, ZDVED-FMK (10 $\mu \mathrm{mol} / \mathrm{L}$; Absin Bioscience), for 10 minutes before incubation with or without glucose $(25 \mathrm{mmol} / \mathrm{L})$ plus collagen $(5 \mu \mathrm{g} / \mathrm{mL})$. Residual platelets and cell debris were used for platelet protein extraction. PMPs were resuspended in MTB for flow cytometry analysis or stored at $-80^{\circ} \mathrm{C}$.

\section{PMPs extraction and flow cytometry analysis}

After treatment, residual platelets and cell debris were removed by using two subsequent centrifugations of $2000 \mathrm{~g}$ for 10 minutes at room temperature. The supernatant was then centrifuged at $18000 \mathrm{~g}$ for 60 minutes to pellet the PMPs[17]. The samples were resuspended with annexin- $\mathrm{V}$ binding buffer, and then incubated with APC-anti-Annexin V and FITC-anti-CD61 (BD Pharmingen) for 30 minutes at $4^{\circ} \mathrm{C}$ in the dark. Next, beads of $0.8 \mu \mathrm{m}$ and $3 \mu \mathrm{m}$ in diameter were used for gating and counting control, respectively. 
$3 \mu \mathrm{m}$ beads $\left(10^{6}\right.$ events) were added to each sample rightly before detection. PMPs were detected as the Annexin $\mathrm{V}^{+} / \mathrm{CD} 1^{+}$particles in the size gate by a FACSCalibur cytometer (BD Biosciences). Analysis was performed using FlowJo (Tree Star Inc.) software. Results were expressed as the number of PMPs from every $10^{8}$ platelets.

\section{Electron microscopy}

Immediately after incubation with glucose $(30 \mathrm{mmol} / \mathrm{L})$ following the background of collagen $(5 \mu \mathrm{g} / \mathrm{mL})$, the platelets suspension was fixed in $2.5 \%$ glutaraldehyde for 2 hours at $4{ }^{\circ} \mathrm{C}$ and then centrifuged at 2000 $\mathrm{g}$ for 5 minutes. The precipitant was washed with MTB and then postfixed in $1 \%$ osmium tetroxide in MTB with sucrose for 2 hours. After dehydration and embedding, the specimens were cut into ultrathin sections and observed by transmission electron microscopy (Leica).

\section{RNA preparation and real-time PCR}

The residual platelets and cell debris were obtained after centrifugation, and washed twice with PBS (pH 7.4). Total RNA from platelets was extracted by TRIzol (TaKaRa). The concentration of RNA was checked by Nanodrop (Thermo). The cDNA was obtained using Primescript ${ }^{\mathrm{TM}}$ RT Master Mix (TaKaRa), and then amplified in a $20 \mu \mathrm{L}$ reaction on $\mathrm{ABI}$ Prism 7300 sequence detection system (Applied Biosystems) using SYBR Premix Ex Taq ${ }^{\mathrm{TM}}$ (TaKaRa). Predesigned primers were purchased from Invitrogen ${ }^{\mathrm{TM}}$ Life Technology and $\beta$-actin served as the housekeeping gene (Table 1). Data were generated from each reaction and normalized to the control group as calculated by the $2^{-\Delta \Delta \mathrm{Ct}}$ method. 


\section{Western blotting}

Total protein from the platelets was extracted using radioimmunoprecipitation assay lysis buffer combined with phenylmethanesulfonyl fluoride and phosphatase inhibitors and quantified using a bicinchoninic acid assay kit (KeyGEN BioTECH). Protein samples were separated by sodium dodecyl sulfatepolyacrylamide gel electrophoresis and then semi-wet transferred onto polyvinylidene fluoride membranes. Non-specific binding sites were blocked with 5\% BSA dissolved in Tris-buffered saline (TBS) containing $0.1 \%$ Tween 20 for 1 hour at room temperature. Membranes were respectively incubated with primary antibodies of anti-ROCK1, anti-caspase-3, anti- myosine phosphatae targeting subunit 1 (MYPT1), anti-p-MYPT1 (Cell Signaling Technology), and anti-GAPDH (Proteintech Group) overnight at $4^{\circ} \mathrm{C}$. Peroxidase-conjugated secondary antibodies were incubated with the membranes for 1 hour. Detection of the bands was performed using chemiluminescent horseradish peroxidase substrate (Merck Millipore) with an ImageQuant LAS 4000 mini acquisition system.

\section{Caspase-3 activity measurement}

The activity of caspase- 3 was measured in the cell lysates of platelets treated as indicated using a caspase3 activity assay kit (Beyotime Biotechnology). Briefly, the cell lysates were incubated with Ac-DEVD$p$ NA substrate, and the absorbance value of the production of $p$ NA at $405 \mathrm{~nm}$ was measured using a spectrophotometer (Thermo Scientific). The results are shown as caspase-3 activity per unit protein.

\section{Statistical analysis}


Data are shown as the mean \pm standard deviation (SD) and analysed using SPSS 19.0 and GraphPad Prism

7.0. Data comparisons between two groups with only one treatment were analysed using $t$-tests. Experiments with multiple treatment groups were assessed by one-way ANOVA, and followed by Student-Newman-Keuls multiple comparison test. $P<0.05$ was considered statistically significant.

\section{Results}

\section{High glucose enhanced PMPs shedding}

The effect of high glucose on shedding of PMPs from platelets was firstly detected by flow cytometry. PMPs were indicated as both annexin V and CD61-positive events that are in the size gate (Figure 1A). As shown in Figure 1B, high glucose $(30 \mathrm{mmol} / \mathrm{L})$ alone did not enhance PMPs generation. Moreover, mannitol, an osmotic pressure control, didn't increase the PMPs release in platelets. Stimulation with the physiological agonist collagen $(5 \mu \mathrm{g} / \mathrm{mL})$ alone induced the release of PMPs compared with the control group. Interestingly, there was a significant increase for the number of PMPs when platelets were stimulated with collagen $(5 \mu \mathrm{g} / \mathrm{mL})$ plus high glucose $(30 \mathrm{mmol} / \mathrm{L})$ compared with collagen stimulation alone. PMPs generation in activated platelets was also confirmed by using transmission electron microscopy which were circle-like particles and about $0.2 \mu \mathrm{m}$ in diameter (Figure 1C). Subsequently, we explored the mechanism of high glucose-induced PMPs generation.

\section{Activation of ROCK1 mediated PMPs generation under the stimulation of high glucose}


Since ROCK plays important role in reorganizing the cytoskeleton, experiments were performed to determine whether ROCK is involved in PMPs generation under the stimulation of high glucose. To gain an insight into the target of PMPs shedding, we firstly measured the mRNA levels of ROCK1 and ROCK2.

The results demonstrated that ROCK1, but not ROCK2, was highly upregulated after stimulation with high glucose and collagen (Figure 2A). To further confirm the target, the protein levels of ROCK1 were also examined by Western blotting. The results were in accordance with the upregulated mRNA levels. Furthermore, the upregulation of ROCK1 was abolished by the selective ROCK inhibitor Y-27632 (Figure 2B). The increased activity of ROCK1, evaluated by the p-MYPT1 to MYPT1 ratio, was also inhibited by Y-27632 in platelets under the high glucose (Figure 2C). The role of ROCK1 in PMPs shedding was then investigated. Y-27632 significantly inhibited the PMPs release induced by high glucose following the background of collagen, while Y-27632 had no effect on the PMPs release from untreated platelets (Figure 2D). These results indicate that ROCK1 plays an important role in high-glucose induced PMPs generation.

\section{Caspase-3 activation played a crucial role in ROCK1 activation-mediated PMPs generation}

It has been well established that ROCK1 is activated by caspase-3, which mediates membrane blebbing. We hypothesized that caspase-3 contribute to high glucose-induced PMPs generation following the background of collagen. As shown in Figure 3A, the relative expression of cleaved-caspase-3/pro-caspase3 was increased in platelets treated with high glucose plus collagen, which was significantly decreased in the presence of Z-DVED-FMK, a specific caspase-3 inhibitor. Meanwhile, high glucose induced an 
elevated caspase-3 activity following the background of collagen compared with the control. The increased caspase-3 activity was blocked by Z-DVED-FMK, on the contrary, Y-27632 had no effect on high glucose-induced caspase-3 activity (Figure 3B). Furthermore, preincubating platelets with Z-DVEDFMK significantly inhibited high glucose-and collagen-induced PMPs release (Figure 3C). Subsequently, the effect of caspase-3 on ROCK1 activation in platelets was studied in the presence of high glucose. The increased expression of ROCK1 in platelets induced by high glucose plus collagen were blocked by ZDVED-FMK, which had no effect on platelets from the control (Figure 3D). More importantly, elevated ROCK1 activity displayed as the ratio of Y-MYPT1/MYPT1 was also abolished by Z-DVED-FMK in platelets treated with high glucose. Taken together, these data suggest that caspase-3 activates ROCK1 and contributes to high glucose-induced PMPs release following the background of low dose of collagen.

\section{Discussion}

This study indicated that high glucose enhanced the PMPs shedding following the background of low dose of collagen. Further analysis showed that aberrant PMPs shedding from activated platelets was caused by activation of ROCK1, but not ROCK2, which primarily due to the cleavage of caspase-3.

Since increased PMPs play an important role in DM-associated macrovascular complications, the mechanism of PMPs generation might be a new therapy target. PMPs shedding occurs after platelet activation, which can be induced by some agonists, such as collagen, thrombin, adenosine diphosphate, and arachidonic acid[18]. DM is typically characterized by chronically elevated blood glucose level. 
Therefore, the mechanism exploration for the most of in vitro study is at the condition of high glucose. High glucose was demonstrated to influence platelet activation[19]. We did not observe an obvious increase in PMPs levels in vitro under the high glucose stimulation alone compared with the normal control or mannitol control. These results could be explained by the fact that glucose is not a physiological agonist of platelets. However, it can increase the intercellular osmotic pressure[20]. Tang et al. demonstrated that platelets were activated by collagen under the stimulation of high glucose compared with normal glucose level, whereas high glucose stimulation had no effect on ADP-induced activation[21]. The flow cytometry analysis and morphological results showed that high glucose increased the PMPs shedding from platelets following the collagen background. This discovery helps us to further explore the potential mechanism underlying PMPs release from activated platelets induced by high glucose.

Based on the platelet microvesiculation process, PMPs generation is accompanied by cytoskeleton rearrangement and phosphatidylserine exposure, which can be used to detect PMPs by flow cytometry. Inhibition of ROCK reversed the endothelial cell-derived microparticles induced by angiotensin II[22]. It has been reported that ROCK activation can alter platelet shape, which is associated with microparticles shedding[23]. ROCK1 and ROCK2 are the two isoforms that perform similar functions in membrane blebbing. Interestingly, our study showed that ROCK1 was upregulated in platelets in response to high glucose following the background of collagen. The function of ROCK1 is mainly manifested in the phosphorylation of the myosin binding subunit MYPT1. Protein expression and activity analysis both confirmed the exactly effects of ROCK1 on the generation of PMPs. In contrast, ROCK2 was the target 
of endothelial microparticle release induced by thrombin[24]. However, ROCK1-deficient mouse platelets expose significantly more phosphatidylserine upon activation by collagen compared with wild-type platelets[25]. These results may be attributed to the different cell types and to the distinct stimuli. Using the ROCK inhibitor Y-27632, we found that the expression and activity levels of ROCK1 were decreased and the PMPs levels were accordingly reduced. These suggest that ROCK1 plays a crucial role in high glucose-induced PMPs shedding folowing the background of collagen.

As we know, to fully activate ROCK1, inhibitory carboxyl terminal domain needs to be removed by caspase-3[14]. Ben Amor et al demonstrated that thrombin activated caspase-3 and 9 in human platelets and increased their active forms by translocating to cytoskeleton[26]. Cohen et al found that PMPs production and platelet caspase-3, 6, and 8 activity levels were increased in diabetic rats[27]. Moreover, Cohen et al demonstrated that the broad-spectrum caspase inhibitor Z-VAD-FMK decreased the phosphatidylserine exposure of platelets in diabetic rats[28]. These findings suggest that caspases have a certain function in platelet activation. Our results showed that cleaved-caspase-3 expression and its activity were upregulated in high glucose-induced activated platelets, which accordingly increased PMPs release. Previous study also showed that PMPs contain caspase-3[29]. These data are in favour of the relationship between caspase-3 and PMPs shedding. The protein expression and activity of caspase- 3 were significantly blocked by Z-DVED-FMK, but not by Y-27632. However, the increased expression and activity levels of ROCK1 in platelets were significantly suppressed by Z-DVED-FMK. Coleman et al demonstrated the involvement of caspase-mediated activation of ROCK1 in membrane blebbing[30]. 
These results indicate that caspase- 3 is on the upstream of ROCK1 and mediates ROCK1 activation in platelets following the condition of high glucose.

In conclusion, we demonstrated that high glucose increased the PMPs shedding. Activation of caspase-3ROCK1 signalling cascade is involved in the PMPs generation. This novel mechanism might provide a potential therapeutic target for preventing the production of circulating PMPs from avtivated platelets in DM.

\section{Acknowledgenments}

None.

\section{Funding}

This work was supported by the National Natural Science Foundation of China (81470957), the Jiangsu Province Six Talent Peaks Project (2015-WSN-002), the Project for Jiangsu Provincial Medical Talent (ZDRCA2016077), the Jiangsu Province Social Development Project (BE2018744), the Fundamental Research Funds for the Central Universities (KYCX18-0182, KYCX17-0169, KYZZ15-0061), and the Jiangsu Province Ordinary University Graduate Research Innovation Project (SJZZ16-004).

\section{References}

[1] Lind M, Svensson AM, Kosiborod M, Gudbjornsdottir S, Pivodic A, Wedel H, et al. Glycemic control and excess mortality in type 1 diabetes. N Engl J Med. 2014;371:1972-82. 
[2] Carracher AM, Marathe PH, Close KL. International Diabetes Federation 2017. J Diabetes. 2018;10:353-6.

[3] Wolf $\mathrm{P}$. The nature and significance of platelet products in human plasma. Br J Haematol. 1967;13(3):269-88.

[4] Boilard E, Nigrovic PA, Larabee K, Watts GF, Coblyn JS, Weinblatt ME, et al. Platelets amplify inflammation in arthritis via collagen-dependent microparticle production. Science. 2010;327(5965):5803.

[5] Risitano A, Beaulieu LM, Vitseva O, Freedman JE. Platelets and platelet-like particles mediate intercellular RNA transfer. Blood. 2012;119(26):6288-95.

[6] S ELA, Mager I, Breakefield XO, Wood MJ. Extracellular vesicles: biology and emerging therapeutic opportunities. Nat Rev Drug Discov. 2013;12(5):347-57.

[7] Sabatier F, Darmon P, Hugel B, Combes V, Sanmarco M, Velut JG, et al. Type 1 and type 2 diabetic patients display different patterns of cellular microparticles. Diabetes. 2002;51(9):2840-5.

[8] Santilli F, Marchisio M, Lanuti P, Boccatonda A, Miscia S, Davi G. Microparticles as new markers of cardiovascular risk in diabetes and beyond. Thromb Haemost. 2016;116(2):220-34.

[9] Badimon L, Suades R, Fuentes E, Palomo I, Padro T. Role of Platelet-Derived Microvesicles As Crosstalk Mediators in Atherothrombosis and Future Pharmacology Targets: A Link between Inflammation, Atherosclerosis, and Thrombosis. Front Pharmacol. 2016;7:293.

[10] Zaldivia MTK, McFadyen JD, Lim B, Wang X, Peter K. Platelet-Derived Microvesicles in Cardiovascular Diseases. Front Cardiovasc Med. 2017;4:74. 
[11] Ferroni P, Basili S, Falco A, Davi G. Platelet activation in type 2 diabetes mellitus. J Thromb Haemost. 2004;2(8):1282-91.

[12] Soma P, Swanepoel AC, du Plooy JN, Mqoco T, Pretorius E. Flow cytometric analysis of platelets type 2 diabetes mellitus reveals 'angry' platelets. Cardiovasc Diabetol. 2016;15:52.

[13] Aoki K, Maeda F, Nagasako T, Mochizuki Y, Uchida S, Ikenouchi J. A RhoA and Rnd3 cycle regulates actin reassembly during membrane blebbing. Proc Natl Acad Sci U S A. 2016;113(13):E186371. Epub 2016/03/16.

[14] Julian L, Olson MF. Rho-associated coiled-coil containing kinases (ROCK): structure, regulation, and functions. Small GTPases. 2014;5:e29846.

[15] Bang J, Jang M, Huh JH, Na JW, Shim M, Carlson BA, et al. Deficiency of the 15-kDa selenoprotein led to cytoskeleton remodeling and non-apoptotic membrane blebbing through a RhoA/ROCK pathway. Biochem Biophys Res Commun. 2015;456(4):884-90.

[16] Duchez AC, Boudreau LH, Naika GS, Bollinger J, Belleannee C, Cloutier N, et al. Platelet microparticles are internalized in neutrophils via the concerted activity of 12-lipoxygenase and secreted phospholipase A2-IIA. Proc Natl Acad Sci U S A. 2015;112(27):E3564-73.

[17] Sisirak V, Sally B, D'Agati V, Martinez-Ortiz W, Ozcakar ZB, David J, et al. Digestion of Chromatin in Apoptotic Cell Microparticles Prevents Autoimmunity. Cell. 2016;166(1):88-101.

[18] Italiano JE, Jr., Mairuhu AT, Flaumenhaft R. Clinical relevance of microparticles from platelets and megakaryocytes. Curr Opin Hematol. 2010;17(6):578-84. 
[19] Yamagishi SI, Edelstein D, Du XL, Brownlee M. Hyperglycemia potentiates collagen-induced platelet activation through mitochondrial superoxide overproduction. Diabetes. 2001;50(6):1491-4.

[20] Sudic D, Razmara M, Forslund M, Ji Q, Hjemdahl P, Li N. High glucose levels enhance platelet activation: involvement of multiple mechanisms. Br J Haematol. 2006;133(3):315-22.

[21] Tang WH, Stitham J, Gleim S, Di Febbo C, Porreca E, Fava C, et al. Glucose and collagen regulate human platelet activity through aldose reductase induction of thromboxane. J Clin Invest. 2011;121(11):4462-76.

[22] Burger D, Montezano AC, Nishigaki N, He Y, Carter A, Touyz RM. Endothelial microparticle formation by angiotensin II is mediated via Ang II receptor type I/NADPH oxidase/ Rho kinase pathways targeted to lipid rafts. Arterioscler Thromb Vasc Biol. 2011;31(8):1898-907.

[23] Aslan JE, McCarty OJ. Rho GTPases in platelet function. J Thromb Haemost. 2013;11(1):35-46.

[24] Sapet C, Simoncini S, Loriod B, Puthier D, Sampol J, Nguyen C, et al. Thrombin-induced endothelial microparticle generation: identification of a novel pathway involving ROCK-II activation by caspase-2. Blood. 2006;108(6):1868-76.

[25] Dasgupta SK, Le A, Haudek SB, Entman ML, Rumbaut RE, Thiagarajan P. Rho associated coiledcoil kinase-1 regulates collagen-induced phosphatidylserine exposure in platelets. PLoS One. 2013;8(12):e84649.

[26] Ben Amor N, Pariente JA, Salido GM, Bartegi A, Rosado JA. Caspases 3 and 9 are translocated to the cytoskeleton and activated by thrombin in human platelets. Evidence for the involvement of PKC and the actin filament polymerization. Cell Signal. 2006;18(8):1252-61. 
[27] Cohen Z, Gonzales RF, Davis-Gorman GF, Copeland JG, McDonagh PF. Thrombin activity and platelet microparticle formation are increased in type 2 diabetic platelets: a potential correlation with caspase activation. Thromb Res. 2002;107(5):217-21.

[28] Cohen Z, Davis-Gorman G, McDonagh PF, Ritter L. Caspase inhibition of platelet activation. Blood Coagul Fibrinolysis. 2008;19(4):305-9.

[29] Boing AN, Hau CM, Sturk A, Nieuwland R. Platelet microparticles contain active caspase 3. Platelets. 2008;19(2):96-103.

[30] Coleman ML, Sahai EA, Yeo M, Bosch M, Dewar A, Olson MF. Membrane blebbing during apoptosis results from caspase-mediated activation of ROCK I. Nat Cell Biol. 2001;3(4):339-45. 
Table 1. Taqman primers for real-time PCR

\begin{tabular}{lll}
\hline Origins & Genes & Taqman primers \\
\hline Rat & ROCK 1 & 5'-GTAAGGAAGGCACAAATGGAG-3' sense \\
& 5'-TGGTTGGGACGTACAGTAAAA-3' antisense \\
Rat & 5OCK 2 & 5'-CAGAGTCAAGAAGCATGTGAGA-3' sense \\
& & 5'-CAGTATAGGCAGTGGACCAGG-3' antisense \\
Rat & 5'-GGCAGTAATCTCCTTCTGCATC-3' antisense \\
\hline
\end{tabular}




\section{Figure Legends}

Figure 1. High glucose enhanced PMPs shedding. Platelets were treated with MTB (5 mmol/L glucose), $25 \mathrm{mmol} / \mathrm{L}$ glucose, $25 \mathrm{mmol} / \mathrm{L}$ mannitol, $5 \mu \mathrm{g} / \mathrm{mL}$ collagen, or $25 \mathrm{mmol} / \mathrm{L}$ glucose plus $5 \mu \mathrm{g} / \mathrm{mL}$ collagen for 30 minutes under steady stirring. (A) Flow cytometry settings for PMPs detection. Beads with diameter of $3 \mu \mathrm{m}$ were used to quantify, and $0.8 \mu \mathrm{m}$ beads were used for setting a size gate. PMPs was detected by flow cytometry as $\mathrm{CD}^{+} 1^{+}$Annexin $\mathrm{V}^{+}$. (B) Flow cytometric analysis of PMPs generated from platelets. The data are shown as the PMPs release $\times 10^{6}$ per $10^{8}$ platelets. Results represent the mean \pm SD. $* * P<0.01$. (C) Transmission electron micrographs of PMPs. Platelets were incubated with $25 \mathrm{mmol} / \mathrm{L}$ glucose in the presence of $5 \mu \mathrm{g} / \mathrm{mL}$ collagen. The black arrow indicates PMPs. Scale bar $=0.2 \mu \mathrm{m}$.

\section{Figure 2. Activation of ROCK1 mediated PMPs generation under the high glucose stimulation. (A)}

Platelets were treated with MTB (Control) or $25 \mathrm{mmol} / \mathrm{L}$ glucose plus $5 \mu \mathrm{g} / \mathrm{mL}$ collagen (HG+Collagen) for 30 minutes. ROCK1 and ROCK2 mRNA expression levels were measured by real-time PCR. Results represent the mean \pm SD. ${ }^{* * *} P<0.001$. (B-D) Platelets were pretreated with the ROCK inhibitor Y-27632 $(10 \mu \mathrm{mol} / \mathrm{L})$ and then stimulated with or without $25 \mathrm{mmol} / \mathrm{L}$ glucose plus $5 \mu \mathrm{g} / \mathrm{mL}$ collagen as described in the section of Materials and Methods. (B) The protein expression levels of ROCK1 were measured by Western blotting. The histograms represent the mean $\pm \mathrm{SD}$ of the ROCK1 expression levels and are normalized to GAPDH. ${ }^{*} P<0.05$. (C) ROCK1 activity in platelets was evaluated by measuring the $p$ MYPT1 substrate levels. The histograms indicate the fold change of p-MYPT1 normalized to MYPT1 shown as the mean $\pm \mathrm{SD} .{ }^{*} P<0.01, * * * P<0.001$. (D) The effects of ROCK1 on PMPs release from 
platelets. PMPs were counted by flow cytometry and the results represent the mean $\pm \mathrm{SD}$. $* * P<0.01$, $* * * P<0.001$

Figure 3. Caspase-3 activation played a crucial role in PMPs generation mediated by ROCK1 activation. Platelets were pretreated with the caspase-3 inhibitor Z-DVED-FMK $(10 \mu \mathrm{mol} / \mathrm{L})$ or ROCK inhibitor Y-27632 $(10 \mu \mathrm{mol} / \mathrm{L})$ and then stimulated with or without $25 \mathrm{mmol} / \mathrm{L}$ glucose following the background of $5 \mu \mathrm{g} / \mathrm{mL}$ collagen for 30 minutes as described in the section of Materials and Methods. (A) Protein expression levels of pro-caspase-3 and cleaved-caspase-3 were measured by Western blotting. The histograms represent the ratio of cleaved-caspase-3 to pro-caspase- 3 . $* * P<0.01$. (B) Caspase-3 activity was determined by chemiluminescence. $* * P<0.01$, $* * * P<0.001$. (C) PMPs release from activated platelets were measured by flow cytometry. $* * P<0.01, * * * P<0.001$. The results represent the mean \pm SD. (D) Protein levels of ROCK1, p-MYPT1 and MYPT1 in platelets were measured by Western blotting. Quantification of ROCK1 expression levels in platelets. Data are shown as the fold change normalized to GAPDH. Quantification of ROCK1 activity is shown as the ratio of p-MYPT1 to MYPT1. ${ }^{*} P<0.05$, $* * * P<0.001$. 
Figure 1.
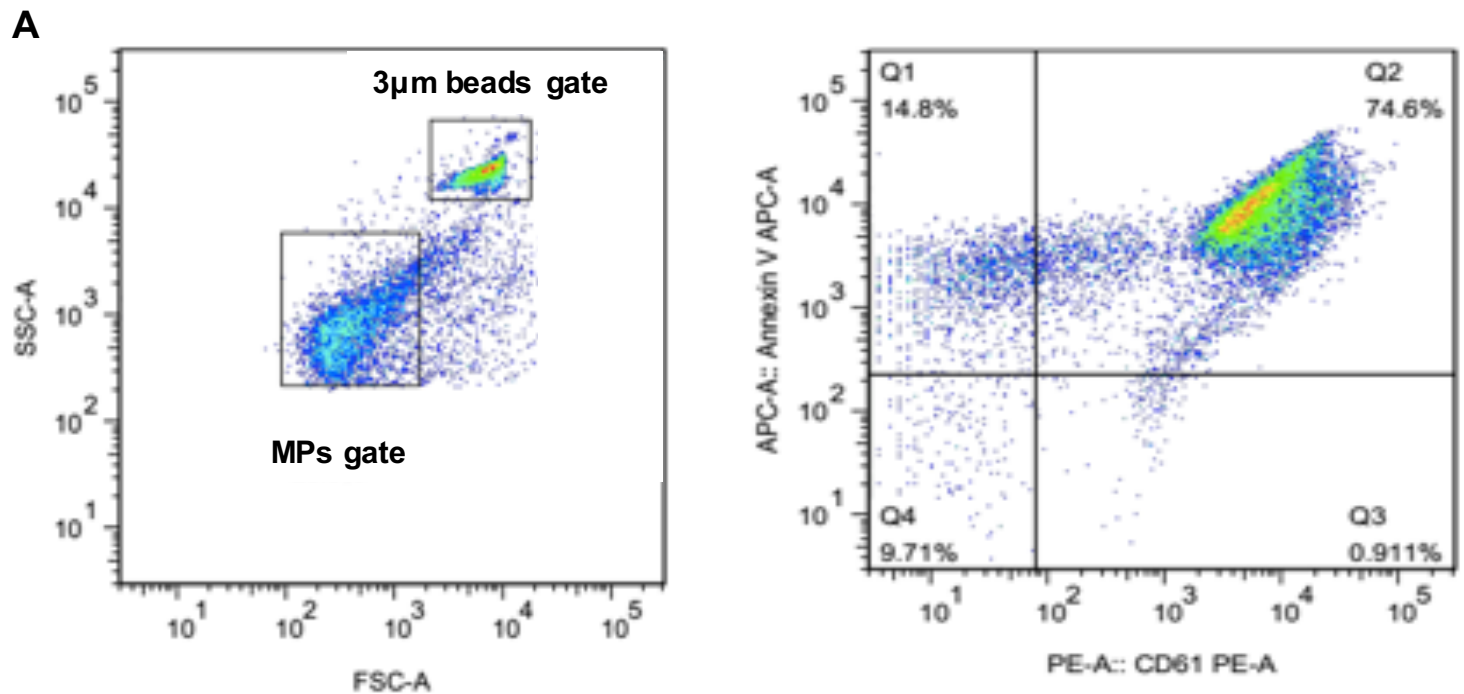

B

C
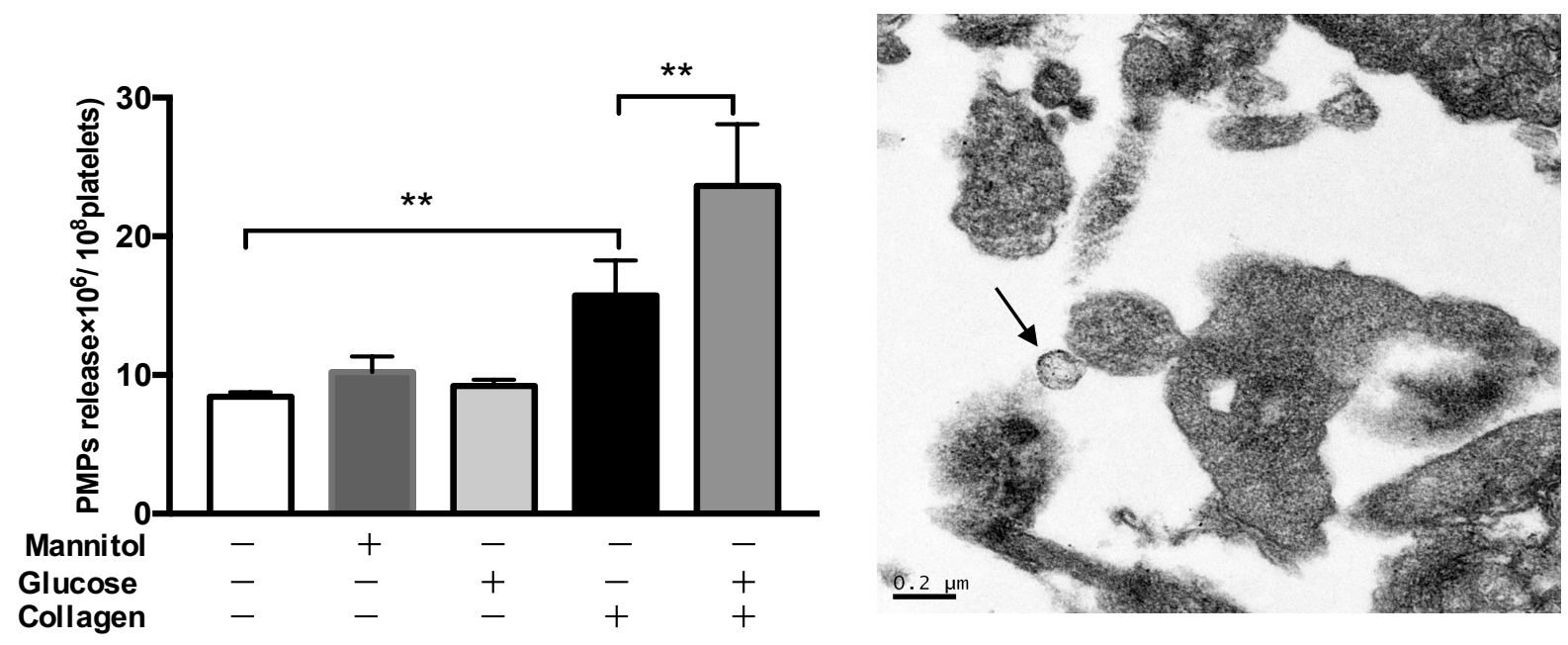
Figure 2.

A

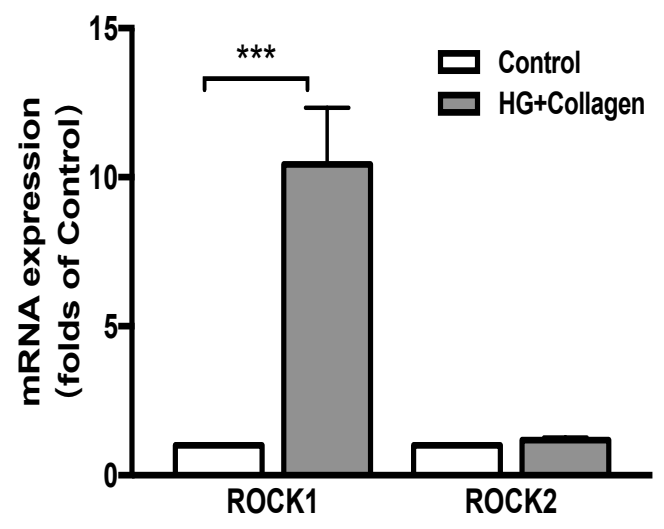

B

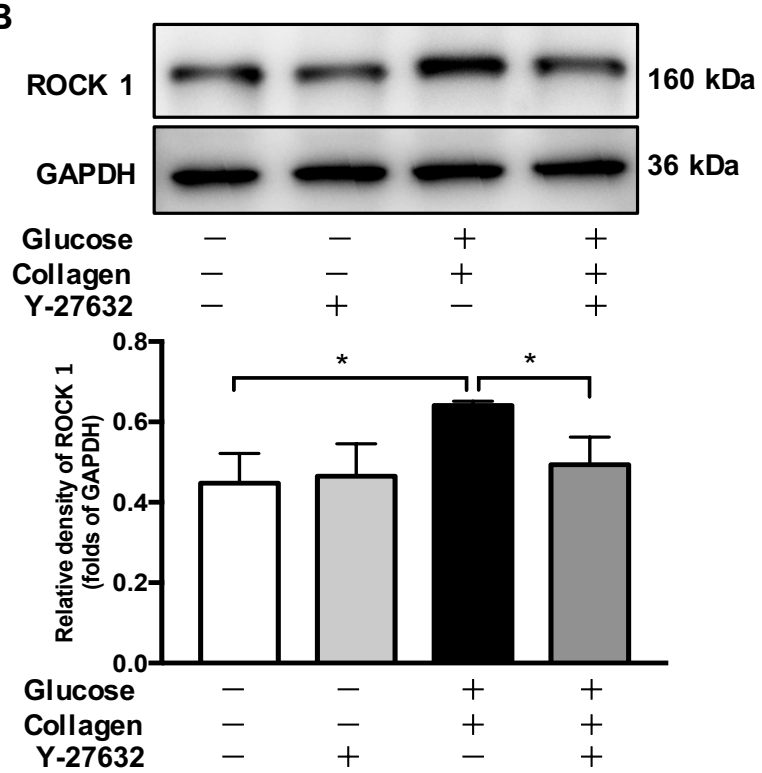

C
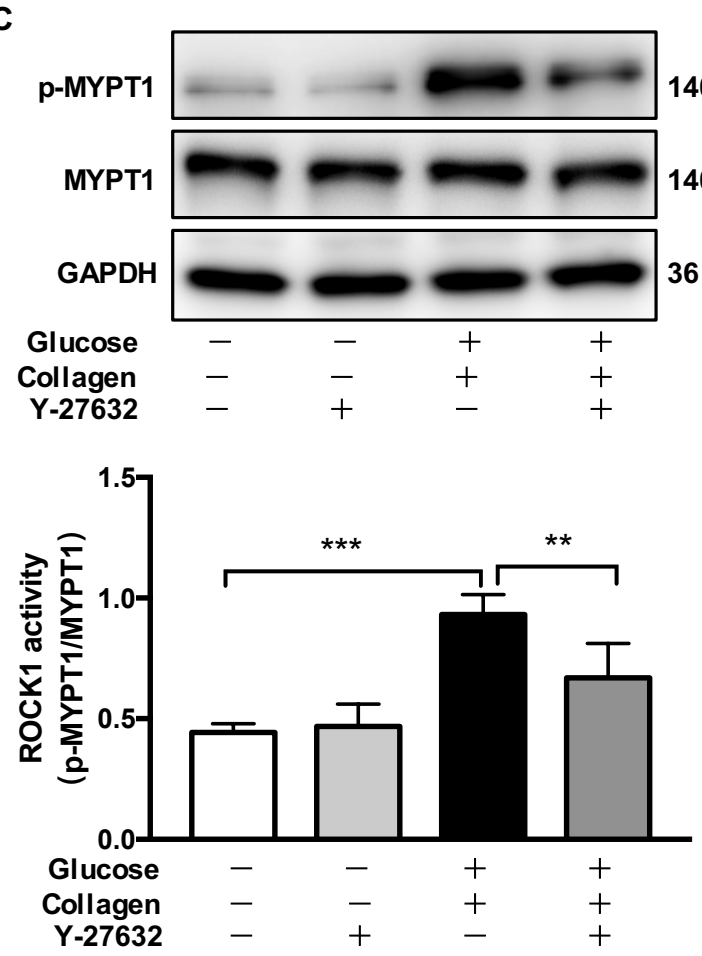

D $140 \mathrm{kDa}$ $140 \mathrm{kDa}$ $36 \mathrm{kDa}$

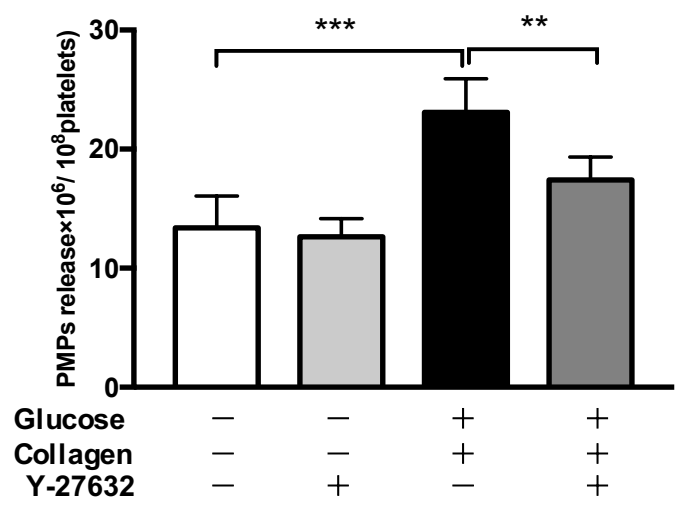


Figure 3.

A

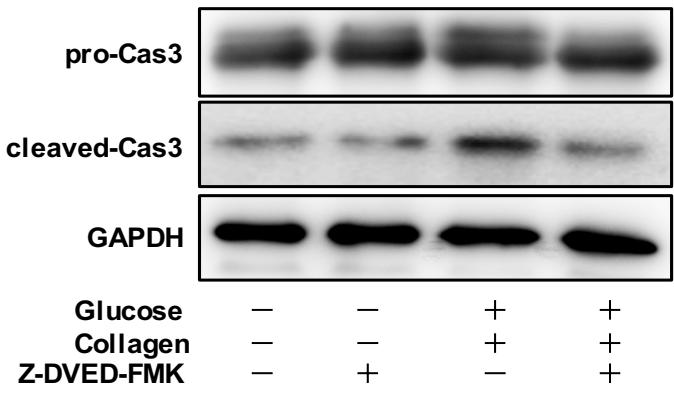

B

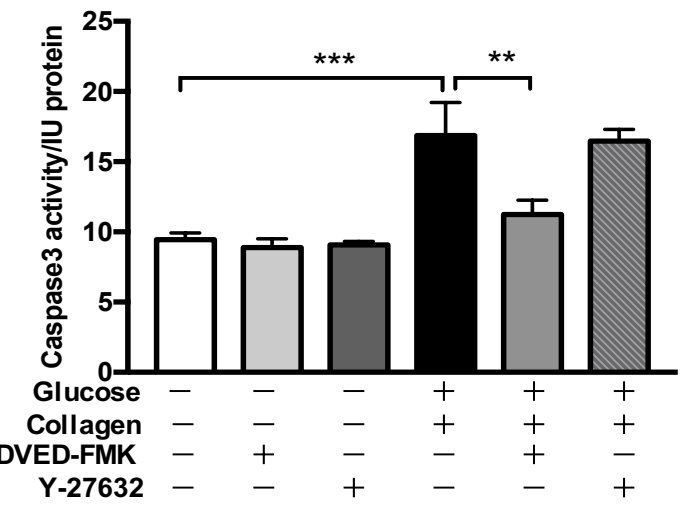

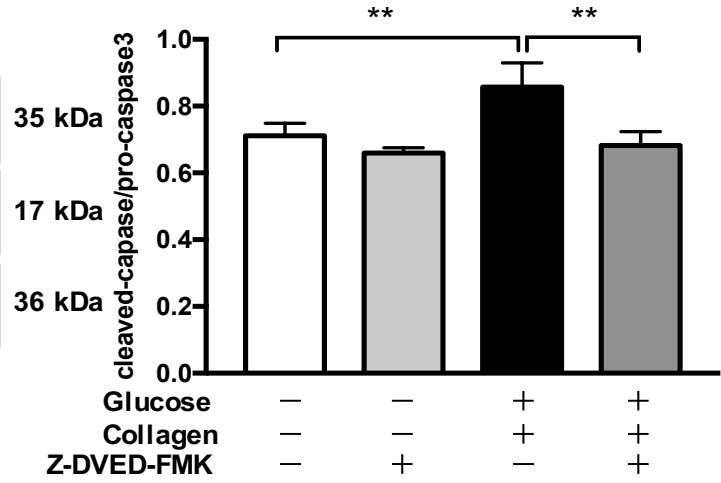

C

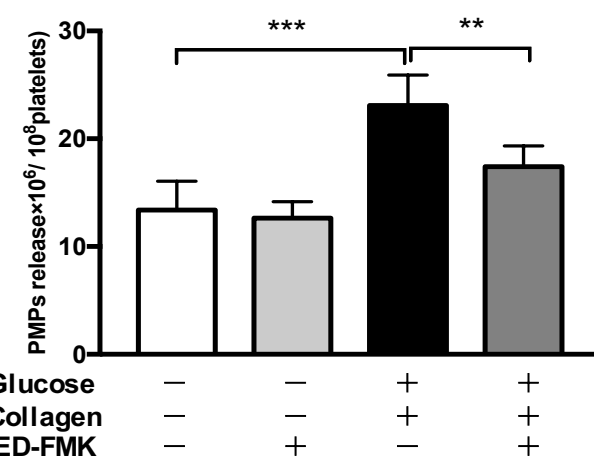

D
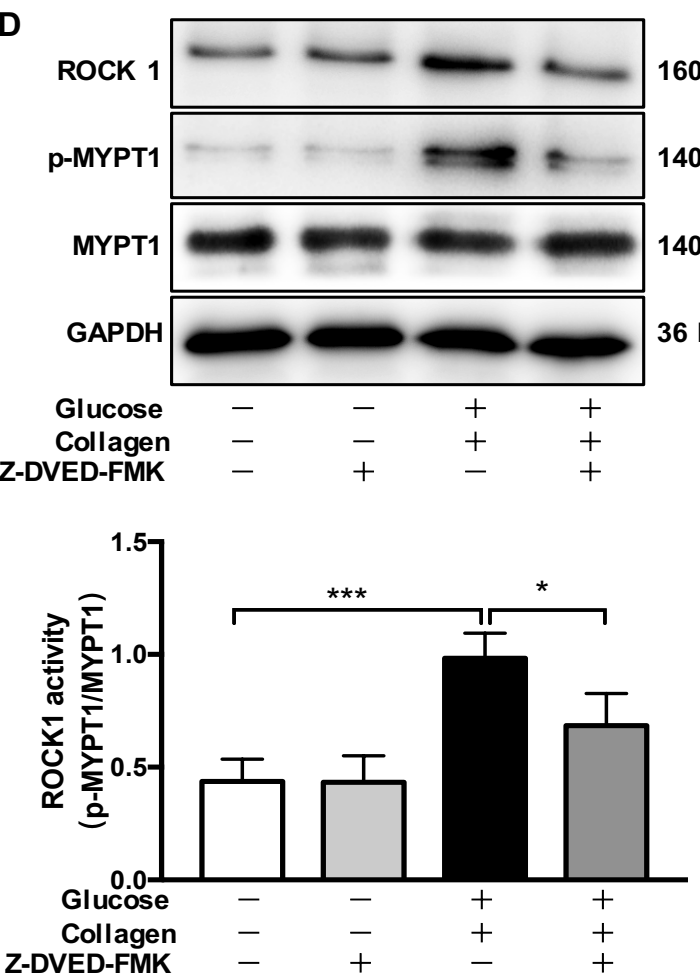

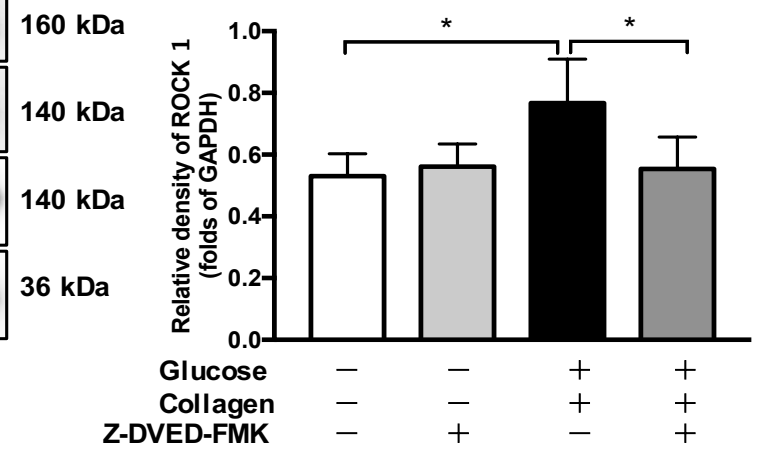


${ }^{*}$ Conflict of Interest

\section{Conflicts of interest}

There is no conflict of interest to be declared by the authors. 